3. Glinoer D. Pregnancy and iodine. Thyroid. 2001;11(5):47181.

4. World Health Organization. Guideline: Fortification of food-grade salt with iodine for the prevention and control of iodine deficiency disorders. Geneva: WHO; 2014.

5. Perú, Ministerio de Salud. Control de la deficiencia de yodo en el Perú, un modelo sostenible. Informe técnico. Lima: MINSA; 1998.

Correspondencia: Eduardo A. Pretell

Dirección: Av. Paseo de la República 3691, Ofic. 401-A. San

Isidro, Lima 27. Perú

Teléfono: (+511) 421-0882

Correoelectrónico:dreapretell@gmail.com

\title{
RÉPLICA: CONCENTRACIONES DE YODO EN ORINA Y EN SAL DE CONSUMO EN MUJERES ENTRE 12 A 49 AÑOS DEL PERÚ
}

\section{REPLY: IODINE CONCENTRATION IN URINE AND SALT CONSUMPTION IN WOMEN BETWEEN 12 TO 49 YEARS OF AGE IN PERU}

\section{Carolina Tarqui-Mamani ${ }^{1,2, a}$}

Sr. Editor. Efectivamente, los resultados del estudio, muestran que la mediana de yoduria en mujeres entre 12 a 49 años fueron $250,4 \mathrm{ug} / \mathrm{L}$, considerados niveles por encima de lo recomendado por la Organización Mundial de la Salud (OMS), que indica una ingesta de yodo más que adecuado y con cierto riesgo de hipertiroidismo inducido por yodo según la OMS ${ }^{(1)}$, pero en ninguna parte del artículo se menciona que la mediana de yoduria es excesiva. Por otra parte, considerar que el nivel de 200-299 $\mu \mathrm{g} / \mathrm{L}$ sea considerado como niveles que permitan asegurar la mayor ingesta de yodo requerida durante la gestación y lactancia, es una propuesta y aún no está aprobada por la OMS.

El artículo presenta la mediana de yoduria en las mujeres entre 12 a 49 años y la mediana de yoduria en gestantes en forma separada, en tanto que en la sección de materiales y métodos se consigna que la mediana de yodo urinario de 100 a $199 \mathrm{ug} / \mathrm{L}$ se consideró nivel adecuado en las mujeres, y la mediana de 150 a 249 ug/L

\footnotetext{
1 Centro Nacional de Alimentación y Nutrición, Instituto Nacional de Salud. Lima, Perú

2 Departamento de Medicina Preventiva y Salud Pública, Facultad de Medicina, Universidad Nacional Mayor de San Marcos. Lima, Perú.

a Doctora en Salud Pública

Recibido: 07-09-15 Aprobado: 16-09-15
}

Citar como: Tarqui-Mamani C. Replica: concentraciones de yodo en orina y en sal de consumo en mujeres entre 12 a 49 años del Perú [carta]. Rev Peru Med Exp Salud Publica. 2015;32(3):613-4. en las gestantes ${ }^{(1)}$, por tanto, no existe distorsión de la "mediana global".

La mediana de yoduria en gestantes fue $274,6 \mathrm{ug} / \mathrm{L}$, y según la OMS los valores adecuados fluctúan entre 150 a $249 \mathrm{ug} / \mathrm{L}$ y que los niveles entre 250 a $499 \mathrm{ug} / \mathrm{L}$ se considera como ingesta de yodo por encima de lo requerido ${ }^{(1)}$, por ello, nuestros resultados muestran que la mediana de yoduria está por encima de lo recomendado por la OMS; al respecto, se entiende, entonces, que los niveles de yodo confieren protección contra el riesgo del daño cerebral fetal, no obstante, los valores están por encima de los niveles recomendados por la OMS y que, eventualmente, pueden ser perjudiciales a la gestante. Asimismo, en la sección de discusión se precisa que la muestra de gestantes es pequeña y los resultados de este grupo deberían ser considerado como una aproximación.

El diseño muestral corresponde a la muestra de la Encuesta Nacional de Hogares (ENAHO) que es probabilística, multietápica, independiente por departamentos, con inferencia a nivel nacional, nacional urbana y rural, departamental, y no se realizó ningún submuestreo, por tanto, las mujeres evaluadas representan a las mujeres residentes en la muestra de hogares de la ENAHO. Cabe precisar que en el marco del convenio entre el Instituto Nacional de Estadística e Informática (INEI) y el Instituto Nacional de Salud (INS), el Centro Nacional de Alimentación y Nutrición (CENAN), puede realizar la evaluación nutricional en toda la muestra de la ENAHO.

La evaluación de la concentración de yodo en sal se realizó mediante análisis cualitativo en todos los hogares evaluados y análisis cuantitativo en cinco departamentos (Cajamarca, Huancavelica, Huánuco, Loreto y San Martín), también se identificó las medianas de yodo según marca de sal la cual se muestra en la Tabla 3. Definitivamente, el análisis cuantitativo es más exacto y preciso, pero en esta evaluación solamente se realizó la evaluación cuantitativa en los departamentos en mención. Al respecto, actualmente se ha realizado la evaluación cuantitativa de yodo en sal en una muestra de escolares de instituciones públicas y privadas de nuestro país, que próximamente será publicada.

Las publicaciones previas en mujeres y escolares muestran medianas de yoduria que están por encima de los niveles recomendados por la OMS, al respecto, Miranda et al. encontraron una mediana de yoduria en escolares de 262,4 ug/L (2); Higa et al., obtuvieron una mediana de yoduria en mujeres en edad fértil de, $266 \mu \mathrm{g} / \mathrm{L}$ (3), y Tarqui et al., mostraron que la yoduria en mujeres entre 12 a 49 años fue 250,4 ug/L. Por otra parte, en países vecinos, como Brasil, se evidenció que existe una 
ingesta excesiva de yodo, y algunos estudios reportaron un incremento de desórdenes tiroideos en Sao Paulo $(4,5)$, por ello, se recomendó disminuir la fortificación de yodo en sal a $20-40 \mathrm{mg} / \mathrm{kg}$. Se sabe, que la yoduria está directamente relacionada con la excreción de yodo a través de la orina (1), entonces, es pertinente publicar los resultados y alertar a la comunidad científica que se podría estar frente un nuevo problema de salud pública debido a la ingesta excesiva de yodo en la población, que muestre la necesidad de analizar la tendencia del hipertiroidismo en nuestro país, antes que se presente o alcance niveles sustanciales.

El Perú, es uno de los países que ha logrado controlar y disminuir los desórdenes por deficiencia de yodo (DDY), sin duda, se reconoce el rol fundamental de la yodación de la sal de consumo considerado la principal intervención que permitiría cubrir los requerimientos de yodo en la población. En nuestro país se ha normado y reglamentado la fortificación de la sal con yodo, cuya vigilancia del cumplimiento de la fortificación está a cargo del CENAN (INS) e incluye la vigilancia de la yoduria en escolares y mujeres, así como la concentración de yodo en sal de consumo en los hogares.

\section{REFERENCIAS BIBLIOGRÁFICAS}

1. World Health Organization. Assessment of iodine deficiency disorders and monitoring their elimination A guide for programme managers. 3rd ed. Geneva: WHO; 2008.

2. Miranda M, Chávez H, Aramburú A, Tarqui-Mamani C; Instituto Nacional de Salud Estado nutricional de yodo en alumnos de escuelas públicas del nivel primario en el Perú, 2009 [Internet]. Lima: INS; 2011 [citado el 23 junio del 2015]. Disponible en: http://www.ins.gob.pe/repositorioaps/0/5/zop/ zona_izquierda_1/INFORME\%20DDI\%202009\%20v2.pdf

3. Higa AM, Miranda M, Campos M, Sánchez JR. Ingesta de sal yodada en hogares y estado nutricional de yodo en mujeres en edad fértil en Perú, 2008. Rev Peru Med Exp Salud Publica. 2010;27(2):195-200.

4. Canaris GJ, Manowitz NR, Mayor G, Ridgway EC. The Colorado thyroid disease prevalence study. Arch Intern Med. 2000;160(4):526-34.

5. Camargo RY, Tomimori EK, Neves SC, G S Rubio I, Galrão AL, Knobel M, et al. Thyroid and the environment: exposure to excessive nutritional iodine increases the prevalence of thyroid disorders in Sao Paulo, Brazil. Eur J Endocrinol. 2008 Sep;159(3):293-9. doi: 10.1530/EJE-08-0192.

Correspondencia: Carolina Tarqui Mamani

Dirección: Instituto Nacional de Salud Calle Tizón y Bueno 276; Jesús María. Lima, Perú

Correo electrónico:carobtm@gmail.com

\section{COMENTARIOS AL ARTÍCULO: EFECTO DE UN SUPLEMENTO NUTRICIONAL A BASE DE LIIPIDOS EN LOS NIVELES DE HEMOGLOBINA E INDICADORES ANTROPOMÉTRICOS EN NIÑOS \\ DE CINCO DISTRITOS DE HUÁNUCO, PERÚ}

\author{
COMMENTS TO THE ARTICLE: \\ EFFECTS OF A LIPID-BASED NUTRIENT \\ SUPPLEMENT ON HEMOGLOBIN LEVELS \\ AND ANTHROPOMETRIC INDICATORS IN \\ CHILDREN FROM FIVE DISTRICTS \\ IN HUANUCO, PERU \\ Giuliana Mercado1,a, Fátima Peña ${ }^{1, a}$, \\ Alonso Soto ${ }^{1, b}$
}

Sr. Editor. Hemos revisado con interés el artículo publicado por Vargas et al. publicado en el último número de su revista (1), el que evalúa el efecto del consumo de un suplemento nutricional a base de lípidos sobre los niveles de hemoglobina, anemia e indicadores antropométricos en niños de seis a once meses de edad de cinco distritos en el departamento de Huánuco. A pesar que los autores de este artículo demuestran sus principales limitaciones, quisiéramos profundizar ciertos temas que nos parecen importantes discutir.

En primer lugar, el objetivo principal del estudio fue evaluar el efecto de estos suplementos, tanto en la anemia como en los indicadores antropométricos. Se evidenció que, a pesar que demuestre una mejoría en los niveles de hemoglobina, existía un efecto negativo sobre los indicadores antropométricos, lo que hace cuestionable su recomendación. A pesar de ello, las conclusiones se centran únicamente en el efecto benéfico sobre la hemoglobina. Esto se conoce en la literatura como reporte selectivo de outcomes ${ }^{(2,3)}$, es decir, centrar la publicación en solo aquellos resultados favorables.

\footnotetext{
Escuela de Medicina, Universidad Peruana de Ciencias Aplicadas. Lima, Perú.

a Estudiante de Medicina, ${ }^{\mathrm{b}}$ médico internista Recibido: 28-07-15 Aprobado: 23-07-15
}

Citar como: Mercado G, Peña F, Soto A. Comentarios al artículo: Efecto de un suplemento nutricional a base de lípidos en los niveles de hemoglobina e indicadores antropométricos en niños de cinco distritos de Huánuco, Perú [carta]. Rev Peru Med Exp Salud Publica. 2015;32(3):614-5. 